Revue internationale P.M.E.

Économie et gestion de la petite et moyenne entreprise

Revure

internationale

PME

\title{
EDI dans la PME et la grande entreprise: similitudes et différences
}

\section{François Bergeron et Louis Raymond}

Volume 9, numéro 1, 1996

URI : https://id.erudit.org/iderudit/1008253ar

DOI : https://doi.org/10.7202/1008253ar

Aller au sommaire du numéro

Éditeur(s)

Presses de l’Université du Québec

ISSN

0776-5436 (imprimé)

1918-9699 (numérique)

Découvrir la revue

Citer cet article

Bergeron, F. \& Raymond, L. (1996). EDI dans la PME et la grande entreprise: similitudes et différences. Revue internationale P.M.E., 9(1), 41-60.

https://doi.org/10.7202/1008253ar
Résumé de l'article

Cette recherche sur l'échange de documents informatisés (EDI) vise à déterminer les avantages que retirent les organisations de cette nouvelle technologie, à comprendre d'où viennent ces avantages et à comparer la situation prévalant dans les PME par rapport à celle retrouvée dans les grandes organisations. Les résultats obtenus indiquent que l'EDI procure aux entreprises divers avantages tels qu'une diminution des coûts administratifs et une amélioration de la rapidité des transactions. Il apparaît aussi que ces avantages dépendent de divers facteurs tels que le degré d'intégration de l'EDI à l'intérieur comme à l'extérieur de l'entreprise et la qualité du processus d'implantation. De nombreuses différences entre les PME et les grandes entreprises ont été observées relativement à leur usage de l’EDIt aux facteurs de succès et aux avantages monétaires qu'elles en retirent. 


\title{
EDI dans la PME et la grande entreprise: similitudes et différences ${ }^{1}$
}

\author{
François BERGERON \\ Université Laval \\ Louis RAYMOND \\ Université du Québec à Trois-Rivières
}

MOTS CLÉS

\section{EDI-Échange de documents informatisés-PME Facteurs de succès - Avantages}

\begin{abstract}
RÉSUMÉ
Cette recherche sur l'échange de documents informatisés (EDI) vise à déterminer les avantages que retirent les organisations de cette nouvelle technologie, à comprendre d'où viennent ces avantages et à comparer la situation prévalant dans les PME par rapport à celle retrouvée dans les grandes organisations. Les résultats obtenus indiquent que l'EDI procure aux entreprises divers avantages tels qu'une diminution des coûts administratifs et une amélioration de la rapidité des transactions. II apparaît aussi que ces avantages dépendent de divers facteurs tels que le degré d'intégration de l'EDI à l'intérieur comme à l'extérieur de l'entreprise et la qualité du processus d'implantation. De nombreuses différences entre les PME et les grandes entreprises ont été observées relativement à leur usage de l'EDI, aux facteurs de succès et aux avantages monétaires qu'elles en retirent.
\end{abstract}

1. Cette recherche a été soutenue financièrement par le Centre francophone de recherche en informatisation des organisations (CEFRIO), le Fonds FCAR du gouvernement du Québec et par le Conseil canadien de l'échange électronique de données. 


\section{ABSTRACT}

An empirical study of 140 organizations was conducted to investigate the benefits organizations could obtain from electronic data interchange (EDI), the success factors of EDI implementation, and the differences among SMEs and large organizations. The results indicate that EDI brings various benefits such as a lowering of administrative costs and improvements in transaction speed and quality of information. These benefits can be obtained dependent upon success factors such as the organizational integration of EDI, the quality of the implementation process and the organizational support. Various differences relative to $E D I$ usage, success factors and monetary benefits were observed between SMEs and larger organizations.

\section{RESUMEN}

La investigacion sobre el intercambio de documentos informatizados (EDI) nos lleva a determinar las ventajas que obtienen las organizaciones con esta nueva tecnología. El objeto es comprender de donde vienen estas ventajas y comparar la situacion predominante en las pequeñas y medianas empresas (PyMEs) con las encontradas en las grandes organizaciones. Los resultados obtenidos indican que el EDI proporciona a las empresas diversas ventajas tales como una disminución de los costos administrativos y una mejoría en la velocidad de la transacciones. Pareciera también que estas ventajas dependen de diversos factores tales como el grado de integración del EDI tanto al interior como al exterior de la empresa y la calidad de los procesos de implementacion o implantacion. Numerosas diferencias entre la PyME y las grandes empresas fueron observadas; estas diferencias son relativas a la utilización des EDI, los factores de éxito y las ventajas monetarias que predominan.

\section{LES AUTEURS}

François Bergeron est professeur titulaire de systèmes d'information à la Faculté des sciences de l'administration de l'Université Laval. II a publié dans des périodiques tels que MIS Quarterly, Journal of Management Information Systems, European Journal of Information Systems, Journal of Small Business Management et Information \& Management. Adresse : Département des systèmes d'information organisationnels, Faculté des sciences de l'administration, Université Laval, Québec, G1K 7P4.

Louis Raymond est professeur titulaire de systèmes d'information au Département des sciences de la gestion et de l'économie de l'Université du Québec à Trois-Rivières. Ses articles ont été publiés dans des revues telles que MIS Quarterly, International Journal of Information Management, Journal of Small Business Management, International Small Business Journal et Entrepreneurship - Theory and Practice. Adresse : Département des sciences de la gestion et de l'économie, Université du Québec à Trois-Rivières, C.P. 500, Trois-Rivières, Québec, G9A 5H7. 


\section{Introduction}

L'échange de documents informatisés (EDI) est une application des nouvelles technologies de l'information permettant à des partenaires d'affaires d'effectuer des transactions commerciales par l'envoi de documents (commandes, factures, etc.) sous forme électronique en les transmettant d'ordinateur à ordinateur au lieu de les écrire et de les poster (Hansen et Hill, 1989; Bergeron et Raymond, 1992b). Présentement, l'EDI est en croissance rapide (Dillon, 1989) et semble promis à un bel avenir dans les entreprises (Straub et Wetherbe, 1989); l'EDI s'inscrit en effet parmi les technologies clés des années 1990 et pourra, selon Russel et Vitale (1988), apporter à l'entreprise un avantage concurrentiel incluant une réduction des coûts et un renforcement des liens commerciaux avec les clients et les fournisseurs.

L'EDI est un phénomène en pleine expansion, et l'on prévoit que le nombre d'entreprises utilisant cette technologie devrait doubler tous les deux ans durant les prochaines années (Sokol, 1989). Actuellement, selon EDI, Spread the Word! (1990), ce marché croît de 35\% par an et environ 1000 entreprises utilisent déjà l'EDI au Canada, incluant un nombre croissant de PME (Bergeron et Raymond, 1992a).

Selon certains chercheurs et de nombreux praticiens, l'implantation de l'EDI est supposée avoir d'importants effets sur le plan économique, organisationnel et technique. Cependant, la plupart des avantages reliés à l'implantation de l'EDI n'ont pas été à ce jour confirmés de manière empirique, et les études réalisées ne sont souvent que des études de cas individuels dont les conclusions ne peuvent que difficilement être généralisées (Banker et Kauffman, 1988 ; Benjamin, De Long et Scott-Morton, 1988 ; Clemons, 1986; Clemons et Row, 1988; Rackoff, Wiseman et Ullrich, 1985; Clemons et Weber, 1990). De plus, plusieurs entreprises, et notamment des PME, ne sont pas véritablement proactives envers l'EDI et ne l'implantent que sous la pression économique de certains de leurs partenaires commerciaux (Bergeron et Raymond, 1992b). Partant de ces constatations, l'objectif de notre étude est triple : déterminer de manière empirique les avantages de l'EDI effectivement retirés par les entreprises qui ont implanté cette technologie, dégager les facteurs de succès qui ont permis à ces entreprises d'obtenir ces avantages et identifier les différences entre les PME et les grandes entreprises.

\section{1. Échange de documents informatisés}

L'expérience des entreprises canadiennes utilisatrices de l'EDI autorise à penser que l'implantation de cette technologie a été associée à certains changements dans l'entreprise, tant sur le plan organisationnel qu'économique. 
D'un point de vue organisationnel, l'implantation de l'EDI requiert le soutien de la direction et l'engagement des principaux gestionnaires dont les activités seront touchées par cette technologie (Harris, 1989; Monczka et Carter, 1988; Shaw, 1988; Evans-Correia, 1989), ainsi que le soutien du personnel des services utilisateurs de l'EDI (Monczka et Carter, 1988). À cet égard, la formation du personnel constitue une étape cruciale de la mise en place de l'EDI (Shaw, 1988; Harris, 1989; Canright, 1988). En effet, tout membre de l'entreprise, qu'il soit cadre, professionnel ou employé de bureau, doit comprendre dans quelle mesure et comment l'EDI affecte son travail. La mise en place de l'EDI entraîne, selon Norris et Waples (1989), les conséquences organisationnelles suivantes: une hausse des exigences de compétence du personnel, une séparation stricte des fonctions, une augmentation du nombre d'autorisations nécessaires, un contrôle accru des moyens de communications et, enfin, une comparaison plus fréquente des données transactionnelles informatisées avec les actifs réels qu'ils représentent. De plus, selon Norris et Waples (1989) et Canright (1988), l'EDI peut accroître l'importance du service des systèmes d'information au sein de l'organisation dans la mesure où il devient un lien indispensable de communication et de coopération entre l'entreprise et ses partenaires.

D'un point de vue économique, l'EDI est censée offrir aux entreprises trois catégories d'avantages (Ferguson et Hill, 1988). Dans un premier temps, l'EDI permet d'épargner du temps et de l'argent en diminuant les coûts des transactions, dans la mesure où celles-ci s'effectuent beaucoup plus rapidement (Canright, 1988; Shaw, 1988). Les coûts d'achat de fournitures, de manipulation et de classement des documents sont aussi réduits dans la mesure où les transactions sont effectuées électroniquement et ne requièrent plus l'utilisation du papier (Canright, 1988; Monczka et Carter, 1988). Il y aussi une baisse des coûts postaux et téléphoniques (Monczka et Carter, 1988) et une baisse du niveau des stocks (Emmelhainz, 1990). Enfin, les coûts de maind'œuvre reliés aux tâches de bureau diminuent dans la mesure où le travail fastidieux de manipulation et de classement des documents en papier est éliminé (Monczka et Carter, 1988; Shaw, 1988).

L'EDI permet aussi à l'entreprise d'améliorer la qualité de ses prestations aux clients et de réduire le temps d'acheminement des transactions et le nombre d'erreurs dans ces transactions. L'EDI permet en outre d'offrir un service plus rapide à la clientèle et de diminuer la durée du cycle de vente (Corley, 1989; Monczka et Carter, 1988; Chain Store Age Executives, 1989; Shaw, 1988). Il permet même d'augmenter le professionnalisme du personnel dans la mesure où l'utilisation de l'EDI exige des procédés de fonctionnement précis et détaillés (Monczka et Carter, 1988). 
Ultimement, l'EDI devrait permettre à l'entreprise de renforcer sa position concurrentielle (Bergeron et Buteau, 1989; Bergeron, Buteau et Raymond, 1991 ; Canright, 1988 ; Norris et Waples, 1989 ; Evans-Correia, 1989). En créant des liens plus étroits entre l'entreprise, ses clients et ses fournisseurs (Osborn, Madnick et Wang, 1990), l'EDI permet à l'entreprise d'ériger des barrières à l'entrée de nouveaux compétiteurs, des barrières à la sortie de ses partenaires commerciaux et des difficultés pour les concurrents d'offrir des services similaires. Par contre, ces avantages concurrentiels peuvent être de courte durée et, dans certains cas, ils représentent plutôt une nécessité concurrentielle pour certaines organisations (Lesjak, 1990) ; c'est le cas en particulier des compagnies aériennes qui doivent maintenant utiliser des systèmes informatisés de réservations de billets d'avion (Clemons, 1991).

La littérature sur l'EDI fait souvent référence aux grandes entreprises (Provigo, Caterpillar, General Motors, etc.), mais elle ne traite que rarement des petites et moyennes entreprises. Or, il a déjà été établi que ces entreprises sont différentes sous maints aspects. Les PME profitent d'avantages uniques tels que leur taille, leur flexibilité, leur capacité à changer rapidement de mission, leur facilité à implanter rapidement des décisions entraînant des changements majeurs, la proximité des marchés et la fidélité de leur clientèle (Julien, Carrière et Hébert, 1988). Par contre, elles ne bénéficient que de ressources limitées, elles n'offrent qu'un nombre limité de produits et de services destinés souvent à un marché très ciblé et elles n'ont que très peu de contrôle sur leur environnement (Dupont, 1986). L'usage des technologies de l'information dans les PME est donc influencé par ces conditions. La pauvreté en ressources humaines, matérielles et financières des PME, leur planification et leur prise de décision intuitive, l'évolution rapide de la firme et sa dépendance envers certaines personnes clés créent des défis importants au développement et à l'implantation de systèmes d'information dans les PME (Raymond, Rivard et Bergeron, 1988 ; Raymond et al., 1990).

Nous constatons donc que les chercheurs et les praticiens attribuent à l'EDI un certain nombre d'avantages bien qu'aucune étude systématique n'ait été menée en vue d'en confirmer l'existence ou d'en analyser la nature. Compte tenu de l'absence de preuves empiriques quant à ces avantages et de l'apparition récente de cette technologie, nous avons étudié plus en détail les effets de l'implantation de l'EDI, ainsi que la présence des différents facteurs pouvant influencer positivement ou négativement cette implantation.

La présente étude tentera donc d'apporter des éléments de réponse aux trois questions suivantes:

- Quels sont les avantages effectivement retirés par les entreprises qui ont implanté l'EDI? 
- Quels sont les facteurs de succès de l'implantation de l'EDI dans ces entreprises?

- Quelles différences observe-t-on entre les PME et les grandes entreprises relativement au contexte d'utilisation de l'EDI, aux avantages et aux facteurs de succès?

\section{Méthodologie de recherche}

\subsection{Modèle et variables de recherche}

Le modèle de recherche relève cinq variables susceptibles d'influer sur les avantages à retirer de l'implantation de l'EDI. Ces variables sont les suivantes : le soutien organisationnel, le processus d'implantation, les procédés de contrôle et le niveau d'intégration; soit quatre variables indépendantes et une variable modératrice, le niveau d'imposition. Les avantages de l'EDI constituent la variable dépendante du modèle.

\subsubsection{Soutien organisationnel}

Cette variable regroupe les éléments qui décrivent les différents acteurs et leur formation préalable à l'EDI dans l'environnement organisationnel étudié (Shaw, 1988 ; Monczka et Carter, 1988; Swanson, 1989). Le premier élément concerne la formation des différentes personnes engagées dans l'EDI incluant la direction, les directeurs de service, le personnel du service des finances, des approvisionnements et le personnel comptable. Le deuxième élément couvre le niveau de soutien qu'apporte la direction au moment de l'implantation. Un troisième élément est relié à la présence d'une structure formelle d'accueil de l'EDI. Le dernier élément porte sur le degré de coopération des différents partenaires commerciaux (clients et fournisseurs) lors de l'implantation de l'EDI.

\subsubsection{Processus d'implantation}

Cette seconde variable regroupe l'ensemble des procédés d'implantation de l'EDI. Le premier élément est relié à la décision même d'implanter l'EDI. Le second aborde les problèmes techniques de l'implantation tels que le développement d'un prototype (fonctions, tests et utilisateurs participants), la réalisation d'un projet pilote (matériels et logiciels sélectionnés), les partenaires commerciaux, les tests réalisés, la documentation, le temps de réponse, le taux d'erreur, les communications réalisées et l'implantation générale du système incluant les transactions affectées et la part de responsabilité des partenaires commerciaux (Jezioro, 1988; Purchasing, 1988; Skagen, 1989). 


\subsubsection{Procédés de contrôle}

Cette troisième variable regroupe les procédés permettant de contrôler l'utilisation de l'EDI. Le premier élément est relié à l'intégrité des données, se mesurant par le nombre et la qualité des opérations de sauvegarde des transactions (nombre d'enregistrements, lieu de stockage des enregistrements). Le deuxième élément est relié au nombre de restrictions à l'utilisation de l'EDI (codes d'accès, mots de passe, temps d'utilisation, utilisateurs autorisés), ainsi qu'au type de transactions et aux spécifications nécessaires à l'identification d'un document (Brown, 1989).

\subsubsection{Niveau d'intégration}

Cette variable a trait à la diffusion de l'EDI à l'intérieur et à l'extérieur de l'organisation. À l'interne, le degré d'intégration se calcule par le nombre de fonctions administratives différentes touchées par l'EDI (commande, livraison, réception, etc.) tandis qu'à l'externe, le degré d'intégration s'évalue par les différents types de partenaires d'affaires utilisant le réseau EDI (clients, fournisseurs, détaillants, institutions financières, etc.).

\subsubsection{Niveau d'imposition}

Le niveau d'imposition concerne l'obligation qu'a eue l'entreprise de participer à un réseau d'EDI. Les entreprises à qui l'on impose l'utilisation de l'EDI subissent plus de contraintes et de changements imprévus. Un lien de dépendance est créé et les avantages retirés peuvent varier en conséquence. Dans certains cas, des organisations ont dû se plier aux volontés d'un partenaire et implanter l'EDI contre leur gré. Des petites et moyennes entreprises, en particulier, ont carrément été désavantagées dans un tel processus (Johnston et Vitale, 1988).

\subsubsection{Avantages de l'EDI}

Cette variable (dépendante) regroupe les différents avantages reliés à l'implantation de l'EDI (Purchasing, 1988). Le premier élément comporte les économies réalisées en matière de coûts (coût des documents, des stocks, de poste et de téléphone). Le deuxième élément touche l'amélioration du service aux partenaires commerciaux (rapidité du service, qualité des transactions). Le troisième élément a trait à la gestion des opérations internes à l'organisation. Enfin, le quatrième élément comprend les retombées en matière d'avantage stratégique que l'entreprise retire de l'implantation de l'EDI (Banker et Kauffman, 1988 ; Benjamin, De Long et Scott-Morton, 1988; Bergeron et Bureau 1989 ; Bergeron, Buteau et Raymond, 1991 ; Bergeron et Raymond, 1992a; 
Crowston et Treacy, 1986; Lederer et Sethi, 1988; Porter, 1985; Porter et Millar, 1985 ; Rackoff, Wiseman et Ullrich, 1985 ; Venkatraman et Zaheer, 1990 ; Vitale, Ives et Beath, 1986 ; Wiseman, 1988). En effet, un nombre croissant d'entreprises utilisent l'échange électronique comme une arme stratégique pour conserver leur clientèle existante et accroître leur part de marché. Le problème actuel reste que l'EDI offre diverses possibilités d'obtenir un avantage concurrentiel, mais lorsque les PME l'implantent sous la pression de clients majeurs, cela peut remettre en cause l'existence même d'un avantage concurrentiel (Clemons, 1986; Clemons et Kimbrough, 1986; Clemons et Row, 1988; Johnston et Vitale, 1988).

\subsection{Hypothèses}

Selon notre modèle de recherche, quatre groupes de variables indépendantes et une variable modératrice vont influer sur les avantages qu'une organisation retire de l'EDI. À partir des considérations précédentes, les sept hypothèses de recherche sont les suivantes:

Hypothèse 1: Plus le soutien organisationnel est favorable, plus les avantages retirés de l'EDI sont élevés.

Hypothèse 2: Plus la qualité du processus d'implantation est élevée, plus les avantages retirés de l'EDI sont élevés.

Hypothèse 3: Plus la qualité des procédés de contrôle est élevée, plus les avantages retirés de l'EDI sont élevés.

Hypothèse 4: Plus l'utilisation de l'EDI est intégrée à l'interne et à l'externe, plus les avantages retirés de l'EDI sont élevés.

Hypothèse 5: Les organisations qui se font imposer l'EDI par leurs partenaires en retirent moins d'avantages.

Hypothèse 6: Le contexte d'implantation de l'EDI est moins favorable dans les PME que dans les grandes entreprises.

Hypothèse 7: Les avantages retirés de l'EDI sont moins élevés dans les PME que dans les grandes entreprises.

\subsection{Collecte des données et mesure des variables}

L'échantillon était composé de 560 entreprises canadiennes membres du Conseil canadien de l'échange électronique de données. Un questionnaire a 
ainsi été envoyé au responsable de la gestion de l'EDI dans chacune de ces entreprises, cette personne ayant été jugée la mieux placée pour fournir l'ensemble des réponses. Un total de 140 questionnaires utilisables ont été retournés pour un taux de réponse de $25 \%$. Les échelles de mesure des variables du modèle étaient de type perceptuel. Différentes échelles ont été utilisées pour mesurer les variables indépendantes du modèle. Pour les variables «soutien organisationnel», "processus d'implantation» et «procédés de contrôle», le répondant devait indiquer jusqu'à quel point les énoncés étaient représentatifs du processus d'implantation de l'EDI dans son organisation. Plusieurs échelles permettaient de mesurer chacune des variables, les échelles étant ancrées en trois points : oui (3), plus ou moins (2) et non (1). La variable «niveau d'imposition» était elle aussi ancrée en trois points selon le même format que les variables précédentes, mais elle ne comportait qu'une seule échelle. Quant à la variable «niveau d'intégration », elle était divisée en deux sous-variables: l'intégration interne et l'intégration externe. L'intégration interne portait sur le nombre de fonctions commerciales différentes traitées par l'EDI dans l'organisation, tandis que l'intégration externe portait sur la variété des types de partenaires avec lesquels l'organisation transigeait.

L'instrument de mesure de la variable dépendante (avantages de l'EDI) portait sur les effets de l'introduction de l'EDI sur les différents aspects de la gestion de l'entreprise. Il couvrait des dimensions telles que les coûts administratifs, la qualité de l'information, la rapidité avec laquelle cette information est véhiculée ainsi que la notion d'avantage stratégique. Cette échelle était de type Likert à 5 points. Les réponses variaient suivant les ancrages suivants : 5 (forte amélioration), 4 (légère amélioration), 3 (aucun changement), 2 (légère détérioration), 1 (forte détérioration). De la même façon que pour les variables indépendantes, les répondants devaient indiquer, en se positionnant sur cette échelle, dans quelle mesure l'implantation de l'EDI avait permis d'obtenir les différents avantages énoncés.

\subsection{Fidélité et validité des échelles de mesure}

Afin de déterminer le nombre et la nature des facteurs présents dans la liste des avantages recensés précédemment, nous avons réalisé une analyse factorielle en composantes principales avec une rotation varimax. La structure retenue, expliquant $61,2 \%$ de la variance, comprenait les cinq facteurs ou catégories d'avantages suivants. Ils sont accompagnés de la valeur alpha de Cronbach et du nombre d'échelles.

- Le facteur «coûts administratifs», relié aux coûts des transactions, des formulaires, de manipulation et de classement de ces formulaires, aux coûts de bureau ainsi qu'au niveau des stocks $(0,84$ et 8$)$. 
- Le facteur «rapidité des transactions», relié au temps requis pour acheminer et traiter une transaction $(0,69$ et 2$)$.

- Le facteur «qualité de l'information», relié aux erreurs de transaction, à la qualité du service à la clientèle, à l'actualité de l'information sur les transactions, à l'accessibilité de l'information ainsi qu'à son exactitude $(0,86$ et 5$)$.

- Le facteur "gestion des opérations », relié à la durée du cycle d'opérations et de prise de décision, ainsi qu'au prix des produits ou services offerts $(0,68$ et 3$)$.

- Le facteur «avantage stratégique», relié à la création de barrières pour les concurrents et de liens plus étroits avec les partenaires commerciaux $(0,50$ et 3$)$.

L'alpha de Cronbach et le nombre d'échelles mesurant les trois premières variables indépendantes sont les suivants: soutien organisationnel $(0,72$ et 11$)$, processus d'implantation $(0,69$ et 12$)$ et procédés de contrôle $(0,75$ et 14$)$. Ces statistiques confirment la fidélité et la validité des mesures.

\section{Résultats}

\subsection{Caractéristiques des répondants}

Les entreprises participantes proviennent de divers secteurs d'activités, mais principalement des secteurs manufacturiers et du transport, la répartition des PME et des grandes entreprises parmi les divers secteurs d'activités étant à peu près identique (tableau 1). L'expérience en EDI varie cependant selon la taille de l'entreprise, car elle était implantée depuis 18 mois dans les PME contrairement à 34 mois dans les grandes entreprises (tableau 2). Nous constatons aussi que l'investissement initial dans l'EDI ainsi que son budget annuel représentent des coûts relativement faibles se chiffrant à $12500 \$$ et $5000 \$$ pour la PME, à $20000 \$$ et $37560 \$$ pour la grande entreprise. Les dépenses en EDI représentent tout de même un coût relatif plus important pour la PME que pour la grande entreprise, car la PME a un chiffre d'affaires médian de 40 millions de dollars comparativement à 250 millions pour la grande entreprise.

Les entreprises utilisent principalement l'EDI pour leurs relations avec les clients et les fournisseurs (tableau 3). Contrairement à la grande entreprise, la PME n'utilise que très peu l'EDI pour communiquer avec les institutions financières. Les deux fonctions les plus répandues de l'EDI sont celles de l'achat de biens et de services et de la facturation (tableau 4). Il existe cependant une différence marquée à ce dernier égard selon la taille de l'entreprise, la PME faisant 
TABleau 1

Secteurs d'activité des organisations $(N=140)$

\begin{tabular}{lrrrr}
\hline Secteurs d'activité & \multicolumn{2}{c}{ PME } & \multicolumn{2}{c}{ GE } \\
& $\boldsymbol{\%}$ & $\mathbf{N}=\mathbf{3 6})$ & \multicolumn{2}{c}{$(\mathbf{N = 1 0 4 )}$} \\
\hline Manufacturier & 25,0 & 9 & 42,3 & $\mathbf{N}$ \\
Distribution & 8,3 & 3 & 8,7 & 9 \\
Transport & 22,2 & 8 & 21,2 & 22 \\
Vente en gros & 13,9 & 5 & 4,8 & 5 \\
Bancaire & 0,0 & 0 & 2,9 & 3 \\
Autres & 30,6 & 11 & 20,2 & 21 \\
\hline
\end{tabular}

1. Chi carré $(\mathrm{p})=8,1(0,15)$

Tableau 2

Caractéristiques des organisations $(N=140)$

\begin{tabular}{lrrrrc}
\hline & \multicolumn{2}{c}{ Médiane } & & \\
& PME & GE & $\mathbf{U}^{\mathbf{1}}$ & $\mathbf{p}^{\mathbf{1}}$ \\
\hline Nombre d'employés & 125 & 1100 & 8,7 & $(0,000)$ \\
Chiffre d'affaires ou budget & & & & \\
(000 \$) & 40000 & 250000 & 5,7 & $(0,000)$ \\
Utilisation de l'EDI (mois) & 18 & 34 & 3,1 & $(0,002)$ \\
Investissement initial dans l'EDI & 12500 & 20000 & 0,8 & $(0,436)$ \\
Budget annuel de l'EDI & 5000 & 37560 & 1,8 & $(0,069)$ \\
\hline
\end{tabular}

1. Valeur $Z$ correspondant au test $U$ de Mann-Whitney pour comparer les médianes.

TABLEAU 3

Types de partenaires d'affaires $(N=140)$

\begin{tabular}{lcccc}
\hline Type de partenaires & $\begin{array}{c}\text { PME } \\
(\mathbf{\%})\end{array}$ & $\begin{array}{c}\text { GE } \\
(\mathbf{\%})\end{array}$ & Phi & p \\
\hline Clients & 66,7 & 72,8 & 0,06 & $(0,48)$ \\
Fournisseurs & 30,6 & 38,8 & 0,08 & $(0,38)$ \\
Distributeurs & 22,2 & 19,4 & 0,03 & $(0,72)$ \\
Compagnies financières & 2,8 & 19,4 & 0,20 & $(0,02)^{*}$ \\
Transporteurs & 13,9 & 14,6 & 0,01 & $(0,92)$ \\
Gouvernement & 16,7 & 8,7 & 0,11 & $(0,19)$ \\
Autres & 11,1 & 12,6 & 0,02 & $(0,81)$ \\
\hline
\end{tabular}

* $\mathrm{p} \leq 0,05$

un usage plus restreint de la fonction de facturation que la grande entreprise. L'écart peut s'expliquer par la possibilité pour la grande entreprise d'exiger que ses partenaires communiquent avec elles par EDI, contrainte que la PME 
peut difficilement imposer à ses partenaires. Les autres activités EDI non mentionnées dans le tableau incluent la communication interne, les données de la paye, la réception et le statut des commandes ainsi que le calendrier des envois.

\section{TABLEAU 4}

Types de transactions effectuées par EDI $(\mathrm{N}=140)$

\begin{tabular}{lclll}
\hline Types de transactions & $\begin{array}{c}\text { PME } \\
(\mathbf{\%})\end{array}$ & $\begin{array}{c}\text { GE } \\
(\mathbf{\%})\end{array}$ & Phi & p \\
\hline Achat de biens et services & 69,4 & 73,8 & 0,04 & $(0,62)$ \\
Facturation & 33,3 & 67,0 & 0,30 & $(0,00)^{*}$ \\
Livraison & 27,8 & 33,0 & 0,05 & $(0,56)$ \\
Transfert de fonds & 5,6 & 14,6 & 0,12 & $(0,16)$ \\
Autres & 27,8 & 21,4 & 0,07 & $(0,43)$ \\
\hline
\end{tabular}

* $\mathrm{p} \leq 0,001$

Au regard de l'utilisation des réseaux spécialisés de communication, nous retrouvons une prépondérance affirmée pour GEISCO, que plus de la moitié des entreprises utilisent (tableau 5). Pourtant, un fait à remarquer est que plusieurs entreprises utilisent non seulement d'autres réseaux spécialisés comme ORDERNET, mais aussi leur propre réseau privé de télécommunications. Enfin, une minorité d'entreprises n'adhèrent à aucun réseau et se servent de lignes téléphoniques commutées pour transmettre leurs données.

En ce qui concerne les protocoles de transmission, nous constatons la prépondérance de la norme ANSI X12 qu'utilisent près des deux tiers des entreprises (tableau 6). Bien que faiblement utilisées en général, les normes UCS et EDIFACT le sont plus par les PME que par les grandes entreprises. Dans l'ensemble, il semble donc que, eu égard à l'uniformisation des standards de transmission, les entreprises semblent se diriger vers une adoption du standard ANSI X12 au Canada plutôt que vers le standard international EDIFACT.

\section{TABLeau 5}

Types de réseau utilisé $(\mathrm{N}=140)$

\begin{tabular}{lcccc}
\hline Réseau utilisé & $\begin{array}{c}\text { PME } \\
(\boldsymbol{\%})\end{array}$ & $\begin{array}{c}\text { GE } \\
(\boldsymbol{\%})\end{array}$ & Phi & p \\
\hline GEISCO (GE) & 44,4 & 57,0 & 0,11 & $(0,20)$ \\
ROUTE COMMERCE & 19,5 & 30,0 & 0,10 & $(0,26)$ \\
(TELECOM) & & & & \\
IBM & 5,6 & 10,0 & 0,07 & $(0,42)$ \\
AUTRES & 36,1 & 35,0 & 0,01 & $(0,90)$ \\
\hline
\end{tabular}


TABLEAU 6

Normes utilisées $(\mathbf{N}=140)$

\begin{tabular}{lcccc}
\hline Normes & $\begin{array}{c}\text { PME } \\
(\mathbf{\%})\end{array}$ & $\begin{array}{c}\text { GE } \\
(\mathbf{\%})\end{array}$ & Phi & p \\
\hline ANSI X12 & 69,4 & 64,1 & 0,05 & $(0,56)$ \\
TDCC & 25,0 & 28,2 & 0,03 & $(0,72)$ \\
UCS & 11,1 & 28,2 & 0,18 & $(0,04)^{*}$ \\
EDIFACT & 11,1 & 2,9 & 0,16 & $(0,05)$ \\
AUTRES & 11,1 & 2,9 & 0,16 & $(0,05)$ \\
\hline
\end{tabular}

* $\quad \mathrm{p} \leq 0,05$

\subsection{Avantages de l'EDI}

Les deux tableaux suivants nous montrent dans quelle mesure les entreprises se sont souciées de leur contexte d'implantation (tableau 7) et nous présentent les avantages qu'elles retirent de l'implantation de l'EDI (tableau 8). Nous constatons que le contexte d'implantation a été de façon générale bien préparé par les entreprises. Il existe cependant trois différences notables entre les PME et les grandes entreprises: dans les PME, les procédures de contrôle sont moins complètes, le nombre d'applications EDI à l'interne est plus limité et les PME sont plus contraintes à implanter l'EDI.

\section{TABleau 7}

Facteurs de succès de l'EDI selon la taille de l'entreprise $(\mathrm{N}=\mathbf{1 4 0})$

\begin{tabular}{lcccc}
\hline & \multicolumn{2}{c}{ Moyenne } & & \\
& PME & GE & T & p \\
& $(\mathbf{N}=\mathbf{3 6})$ & $(\mathbf{N = 1 0 4 )}$ & & \\
\hline Soutien organisationnel $^{1}$ & 2,48 & 2,51 & 0,4 & $(0,69)$ \\
Processus d'implantation $^{1}$ & 2,34 & 2,46 & 1,3 & $(0,21)$ \\
Procédés de contrôle $^{1}$ & 2,31 & 2,48 & 2,1 & $(0,04)^{*}$ \\
Intégration interne $^{2}$ & 1,64 & 2,08 & 2,5 & $(0,01)^{* *}$ \\
Intégration externe $^{3}$ & 1,64 & 1,85 & 1,1 & $(0,27)$ \\
Libre choix $^{4}$ & 2,01 & 2,30 & 1,7 & $(0,09)^{*}$ \\
\hline
\end{tabular}

* $\mathrm{p} \leq 0,05 ; * * \mathrm{p} \leq 0,01$

1. $1:$ non $; 2$ : plus ou moins ; 3 : oui.

2. Nombre de fonctions différentes.

3. Nombre de partenaires diffférents.

4. $1:$ non $; 2:$ plus ou moins $; 3$ : oui. 


\section{TABLEAU 8}

Avantages de l'EDI selon la taille de l'entreprise $(\mathbf{N}=140)$

\begin{tabular}{|c|c|c|c|c|}
\hline \multicolumn{5}{|c|}{ Moyenne ${ }^{1}$} \\
\hline & $\begin{array}{c}\text { PME } \\
(\mathbf{N}=36)\end{array}$ & $\begin{array}{c}\text { GE } \\
(\mathbf{N}=\mathbf{1 0 4})\end{array}$ & $\mathbf{T}$ & $\mathbf{p}$ \\
\hline Coûts administratifs & 3,23 & 3,41 & 1,7 & $(0,09)^{*}$ \\
\hline Rapidité des transactions & 3,50 & 3,61 & 0,5 & $(0,61)$ \\
\hline Qualité de l'information & 3,57 & 3,74 & 1,2 & $(0,24)$ \\
\hline Gestion des opérations & 3,14 & 3,25 & 1,4 & $(0,17)$ \\
\hline Avantage stratégique & 3,24 & 3,23 & $-0,1$ & $(0,91)$ \\
\hline Avantage global & 3,34 & 3,46 & 1,5 & $(0,15)$ \\
\hline
\end{tabular}

Quant au type d'avantages obtenus par les firmes (tableau 8), l'examen des moyennes nous permet de les ordonner. Les avantages les plus importants de l'EDI concernent d'abord la qualité de l'information et la rapidité des transactions. Vient ensuite la réduction des coûts administratifs, alors que l'amélioration de la gestion des opérations et l'obtention d'un avantage stratégique sont moins importants, c'est-à-dire que c'est à ce chapitre que les améliorations dues à l'EDI ont été les moindres. Même si les scores moyens sont assez proches du point central (aucun changement), nous pouvons remarquer que certaines entreprises perçoivent obtenir d'importants avantages (significativement différents du point neutre). Les PME diffèrent des grandes entreprises sur un seul point : bien que positifs, leurs gains relativement aux coûts administratifs demeurent moins élevés que dans la grande entreprise. Si nous considérons le facteur coût comme étant le plus représentatif de la rentabilité de l'EDI pour l'entreprise, il est possible de conclure que l'EDI est moins rentable pour la PME que pour la grande entreprise.

\subsection{Relations entre les facteurs de succès et les avantages}

Nous constatons donc que l'EDI apporte des avantages aux entreprises qui l'ont implanté, et que, dans certain cas, ces avantages peuvent être importants. Le tableau 9 identifie les raisons des fluctuations des résultats entre les entreprises. On y retrouve les coefficients de corrélation entre le contexte d'implantation et les avantages retirés par les entreprises. 
Tableau 9

Corrélations d'ordre zéro entre les variables indépendantes et les variables dépendantes $(N=140)$

\begin{tabular}{lccccc}
\hline & $\begin{array}{c}\text { Soutien } \\
\text { organisa- } \\
\text { tionnel }\end{array}$ & $\begin{array}{c}\text { Processus } \\
\text { d'implan- } \\
\text { tation }\end{array}$ & $\begin{array}{c}\text { Procédés } \\
\text { de } \\
\text { contrôle }\end{array}$ & $\begin{array}{c}\text { Inté- } \\
\text { gration } \\
\text { interne }\end{array}$ & $\begin{array}{c}\text { Inté- } \\
\text { gration } \\
\text { externe }\end{array}$ \\
\hline Coûts administratifs & $0,30^{* * *}$ & $0,30 * * *$ & $0,31 * * *$ & $0,35 * * *$ & $0,29 * * *$ \\
Rapidité de transaction & 0,12 & $0,21 * *$ & $0,20 * *$ & 0,10 & $0,20 * *$ \\
Qualité de l'information & 0,13 & $0,15 *$ & 0,04 & 0,10 & 0,10 \\
Gestion des opérations & $0,18^{*}$ & 0,10 & 0,11 & 0,02 & 0,10 \\
Avantages stratégiques & $-0,05$ & $-0,21 * *$ & $-0,11$ & $0,21 * *$ & $0,21 * *$ \\
Avantage global & $0,26 * * *$ & $0,25 * * *$ & $0,21 * *$ & $0,28 * * *$ & $0,29 * * *$ \\
\hline
\end{tabular}

$* \mathrm{p} \leq 0,05 ; * * \mathrm{p} \leq 0,01 ; * * * \mathrm{p} \leq 0,001$

Il existe une corrélation significative entre les avantages obtenus au regard des coûts administratifs et chacune des variables indépendantes du modèle de recherche. Cela signifie donc qu'une réduction des coûts administratifs a été observée dans les entreprises qui ont porté attention au soutien organisationnel, au processus d'implantation et aux procédés de contrôle, qui utilisent l'EDI pour un plus grand nombre de fonctions commerciales et qui transigent avec plusieurs types de partenaires.

On retrouve aussi des corrélations significatives entre les avantages obtenus au regard de la rapidité des transactions et du processus d'implantation, des procédés de contrôle et de l'intégration interne. Cependant, il n'a pu être démontré que le soutien organisationnel ou l'intégration externe exerçait une influence sur la rapidité avec laquelle les transactions étaient effectuées. En outre, le soutien organisationnel de l'EDI semble avoir un effet positif sur l'amélioration de la gestion des opérations. On notera qu'une corrélation négative entre la démarche d'implantation et l'obtention d'un avantage stratégique a été observée. Il est possible qu'une entreprise qui suit une démarche d'implantation très rigoureuse allonge la période d'implantation à un point tel que cela permet à ses concurrents de l'imiter et même de la devancer à cet égard. Enfin, des corrélations positives ont été observées entre les niveaux d'intégration interne et externe et l'obtention d'un avantage stratégique. Les résultats obtenus montrent que le soutien organisationnel, le processus d'implantation, les procédés de contrôle et l'intégration interne et externe sont déterminants pour l'ensemble des avantages à retirer de l'EDI. Plus précisément, ces variables sont prédictrices d'une réduction des coûts administratifs et de la rapidité des transactions. Elles favorisent aussi l'obtention d'un avantage stratégique et l'amélioration de la gestion des opérations. Il semblerait donc indiqué de porter une attention particulière à ces groupes de variables pour maximiser les avantages procurés par l'EDI. 


\section{TABLEAU 10}

Valeur moyenne des avantages de l'EDI

selon le niveau d'imposition $(N=140)$

\begin{tabular}{lccccc}
\hline & $\begin{array}{c}\text { Imposé } \\
\text { par le } \\
\text { partenaire } \\
(\mathbf{N = 8 9 )}\end{array}$ & $\begin{array}{c}\text { Écart } \\
\text { type }\end{array}$ & $\begin{array}{c}\text { Non imposé } \\
\text { par le } \\
\text { partenaire } \\
(\mathbf{N = 5 1 )}\end{array}$ & $\begin{array}{c}\text { Écart } \\
\text { type }\end{array}$ & $\begin{array}{c}\text { Test T } \\
\text { bicaudal } \\
\text { (sig) }\end{array}$ \\
\hline Coûts administratifs & 3,22 & 0,47 & 3,61 & 0,57 & $0,000^{* * *}$ \\
Rapidité de transaction & 3,54 & 1,07 & 3,67 & 1,11 & 0,512 \\
Qualité de l'information & 3,63 & 0,65 & 3,80 & 0,88 & 0,225 \\
Gestion des opérations & 3,15 & 0,38 & 3,34 & 0,58 & $0,044^{*}$ \\
Avantages stratégiques & 3,20 & 0,40 & 3,30 & 0,52 & 0,233 \\
Avantage global & 3,34 & 0,37 & 3,59 & 0,45 & $0,001^{* * *}$ \\
\hline
\end{tabular}

$* \mathrm{p} \leq 0,05 ; * * \mathrm{p} \leq 0,01 ; * * * \mathrm{p} \leq 0,001$

L'importance du niveau d'imposition de l'EDI est appuyée par les données du tableau 10. Les valeurs moyennes des avantages diffèrent significativement selon que l'EDI a été imposé par les partenaires ou non. En effet, on remarque que les avantages obtenus sont de façon générale plus faibles dans les entreprises à qui l'on a imposé l'EDI. Plus précisément, ces entreprises retireront nettement moins d'avantages au regard des coûts administratifs et de la gestion des opérations. Une analyse des corrélations partielles entre les facteurs de succès et les avantages, tenant compte du niveau d'imposition, a permis de constater que la direction et l'ampleur des relations expliquées ci-dessus entre les facteurs de succès et les avantages à retirer de l'EDI demeurent les mêmes tant chez les entreprises qui ont été contraintes d'introduire l'EDI que chez celles qui l'ont adopté volontairement. Seuls les avantages varient selon le niveau d'imposition (tableau 10), confirmant d'autant l'importance de ces variables en tant que facteurs expliquant le succès de l'EDI. La même analyse de corrélations partielles, tenant compte à la fois du niveau d'imposition et de la taille de l'entreprise, a aussi révélé que la direction et l'ampleur des relations expliquées demeuraient les mêmes tant pour la PME que pour la grande entreprise.

\section{Conclusion}

Le but de la recherche était de déterminer les facteurs de succès et les avantages retirés de l'utilisation de l'EDI et de comparer la situation de la PME à celle de la grande entreprise. Les résultats montrent que les entreprises profitent en général de cinq catégories d'avantages reliés à l'utilisation de cette technologie, à savoir: une réduction des coûts administratifs, une plus grande rapidité de traitement des transactions, une amélioration de la qualité de l'information transigée, une amélioration de la gestion opérationnelle et l'obtention d'un 
avantage stratégique. De façon générale, ces avantages ont été relevés dans les entreprises qui ont soutenu l'implantation de l'EDI dans l'organisation, qui ont établi des procédés de contrôle, qui ont intégré l'EDI autant à l'intérieur qu'à l'extérieur de l'organisation, qui ont été attentives à son processus d'implantation et qui n'ont pas été contraintes par leurs partenaires d'affaires de l'adopter.

Par opposition aux grandes entreprises, les PME qui utilisent l'EDI se distinguent sous différents points. Les PME ont un profil de partenaires d'affaires différent, principalement en ce qui a trait au peu de relations EDI qu'elles entretiennent avec les compagnies financières. Elles font aussi une utilisation moins grande de cette technologie pour la facturation des biens et services rendus. Quant aux normes EDI utilisées, elles font un plus grand usage d'EDIFACT, mais utilisent moins les normes UCS. Au chapitre des facteurs permettant aux entreprises de retirer des avantages de l'usage de l'EDI, les PME sont plus faibles que les grandes entreprises sur trois points : les procédés de contrôle assurant la sécurité des données sont moins développés; le nombre de fonctions internes utilisant l'EDI est moindre et les PME sont plus souvent contraintes par leurs partenaires commerciaux à adopter la technologie EDI. Or, contrairement à ce qui avait été prévu, les PME ont pu obtenir autant d'avantages que les grandes entreprises, sauf en ce qui a trait à la diminution des coûts. L'EDI est donc une technologie qui peut être profitable pour les PME dans la mesure où ces entreprises porteront une plus grande attention au contexte d'implantation.

\section{Bibliographie}

BANKER, R.D. et R.J. KAUFFMAN (1988), «Strategic contributions of information technology: an empirical study of ATM Networks », Comptes rendus de la $9^{e}$ International Conference on Information Systems, Minneapolis, Minnesota, 30 novembre-3 décembre, p. 141-150.

BENJAMIN, R.I., D.W. DE LONG et M.S. SCOTT-MORTON (1988), «The realities of electronic data interchange: How much competitive advantage?», CISR, Document de travail $n^{\circ} 166$, Sloan School of Management, MIT, Massachusetts, janvier.

Bergeron, F. et C. Buteau (1989), «Devancer la concurrence par les systèmes d'information », Revue Internationale PME, vol. 1, n 3, p. 295-307.

Bergeron, F., C. Buteau et L. RAYMond (1991), «Identification of strategic information systems opportunities: applying and comparing two methodologies », MIS Quarterly, vol. 15, n 1, mars.

Bergeron, F. et L. RAYMOND (1992a), «Planning of information systems to gain a competitive edge», Journal of Small Business Management, vol. 30, $\mathrm{n}^{\circ} 1$, janvier, p. 21-26. 
BERGERON, F. et L. RAYMOND (1992b), «The advantages of electronic data interchange », Data Base, vol. 23, no 4, p. 19-31.

Brown, B. (1989), «VANs tell users of EDI legal problems », Network World, vol. $17, \mathrm{n}^{\mathrm{o}} 17,1^{\mathrm{er}}$ mai, p. 11-15.

CANRIGHT, C. (1988), «Seizing the electronic information advantage », Business Marketing, vol. 73, $\mathrm{n}^{0} 1$, janvier, p. 81-86.

Chain Store Age Executives (1989), «Vendors slow to implement quick response », vol. 65, $\mathrm{n}^{\circ} 1$, janvier, p. 107-111.

Clemons, E.K. (1986), «Information systems for sustainable competitive advantage », Information and Management, vol. 11, $\mathrm{n}^{\circ}$ 3, octobre, p. 131-136.

Clemons, E.K. et S.O. Kimbrough (1986), «Information systems, telecommunications and their effects on industrial organization », Comptes rendus de la 7 e International Conference on Information Systems, San Diego, California, 15-17 décembre, p. 99-108.

Clemons, E.K. et M. Row (1988), «McKesson Drug Company : a case study of Economost-a strategic information system », Journal of Management Information Systems, vol. 5, $\mathrm{n}^{\circ} 1$, p. 36-50.

Clemons, E.K. et B.W. WeBer (1990), «London's Big Bang: a case study », Journal of Management Information Systems, vol. 6, $\mathrm{n}^{\circ} 4$, p. 41-60.

Clemons, E.K (1991), «Evaluation of strategic investments in information technology », Communications of the ACM, vol. 34, $\mathrm{n}^{0} 1$, janvier, p. 22-36.

CORLEY, R. (1989), "Networking hospital computers-essential to survival», Federation of American Health Systems Review, vol. 22, nº 1, janvier-février, p. $65-71$.

Crowston, K. et M.E. Treacy (1986), «Assessing the impact of information technology on enterprise level performance », Comptes rendus de la $7^{e}$ International Conference on Information Systems, San Diego, California, 15-17 décembre, p. 299-310.

Dillon, T. (1989), «Purchasing is EDI big winner», Purchasing World, vol. 33, $\mathrm{n}^{\circ} 4$, avril, p. 32-34.

Dupont, C. (1986), «Les PME face aux mégatrends », Revue Française de Gestion, janvier-février, p. 96-105.

EDI SPREAD THE WORD ! (1990), EDI Yellow Pages International, édition V, janvier, EDI Spread the Word!, P.O. Box 811366, Dallas, Texas 75381.

Emmelhainz, M.A. (1990), Electronic Data Interchange, New York, Van Nostrand Reinhold.

Evans-Correia, K. (1989), «EDI : the future frontier », Purchasing, vol. 106, $\mathrm{n}^{\circ} 3$, 23 février, p. 44-47. 
Ferguson, D. et N. Hill (1988), «Missing the boat», Business Credit, vol. 90, $\mathrm{n}^{\circ} 11$, décembre, p. 21-24.

HANSEN, J. et N. HiLl (1989), "Control and audit of electronic data interchange», MIS Quarterly, vol. 13, n 4, décembre, p. 403-414.

HARRIS, K. (1989), «How to start an electronic data interchange program », Electrical World, février, p. 33-34.

Jezioro, A. (1988), «Computer talk», Mortgage Banking, vol. 48, $\mathrm{n}^{\circ}$ 6, mars, p. $36-44$.

Johnston, H.R. et M.R. Vitale (1988), «Creating competitive advantage with interorganizational information systems », MIS Quarterly, vol. 12, n 2, juin, p. 153-165.

Julien, P.A., J.B. CARrière et L. HÉBERT (1988), «Le rythme de pénétration des nouvelles technologies dans les PME manufacturières québécoises », Revue Internationale PME, vol. 1, $\mathrm{n}^{\circ} 2$, p. 193-222.

LEDERER, A.L. et V. SETHI (1988), «The implementation of strategic information systems planning methodologies », MIS Quarterly, septembre, p. 445-462.

LESJAK, D. (1990), «Interorganizational information system as a foundation for electronic data interchange which contributes to organization competitiveness », Comptes rendus de la $3^{e}$ Electronic Data Interchange Conference, J. Gricar (éd.), Bled, Yugoslavia, 4-5 juin, p. 121-132.

MONCZKA, R.M. et J.R. CARTER (1988), «Implementing electronic data interchange », Journal of Purchasing and Materials Management, p. 1-9.

NORRIS, D. et E. WAPLES (1989), «Control of electronic data interchange systems », Journal of Systems Management, vol. 40, $\mathrm{n}^{\circ} 3$, mars, p. 21-25.

Osborn, C.S., S.E. MAdNICK et Y.E. WANG (1990), "Strategic alliance for composite IS », Information and Management, vol. 6, n ${ }^{0} 3$, p. 99-118.

Porter, M.E. (1985), Competitive Advantage: Creating and Sustaining Superior Performance, New York, Free Press.

PorTER, M.E. et V.E. MiLlar (1985), « How information gives you competitive advantage », Harvard Business Review, vol. 63, n 4, juillet-août, p. 149-160.

PURCHASING (1988), «Paperless purchasing», vol. 105, n 1, juillet, p. 98b3, b7.

RACKOFF, N., C. Wiseman et W.A. UllRICH (1985), «Information systems for competitive advantage : implementation of a planning process », MIS Quarterly, vol. $9, \mathrm{n}^{\circ} 4$, décembre, p. 185-294.

RAYMOND, L., S. RIVARD et F. BERGERON (1988), L'informatisation dans les PME: 12 cas types, Québec, Institut de recherches politiques et Presses de l'Université Laval, 282 p. 
RAYmond, L., F. Bergeron, L. Gingras et S. Rivard (1990), «Problématique de l'informatisation des PME », Technologies de l'Information et Société, vol. 3, $\mathrm{n}^{\circ} 1$, p. 131-148.

Russel, J. et M. Vitale (1988), «Creating competitive advantage with interorganisational information systems », MIS Quarterly, juin, p. 153-165.

SHAW, J. (1988), «Planning for EDI », Business Credit, vol. 90, n 11, décembre, p. 18-20, 57.

SKAGEN, A. (1989), « Nurturing relationships, enhancing quality with electronic data interchange », Management Review, vol. 78, $\mathrm{n}^{\circ}$ 2, février, p. 28-32.

SoKol, P.K. (1989), EDI: The Competitive Edge, New York, McGraw-Hill.

Straub, W. et J. Wetherbe (1989), «Information technologies for the 1990s: an organisational impact perspective», Communications of the ACM, vol. 32, $n^{0} 11$, novembre, p. 1328-1339.

Swanson, B. (1989), Information Systems Implementation, Homewood, Illinois, Irwin.

VenkatramanN, N. et A. ZaheER (1990), «Electronic integration and strategic advantage : a quasi experimental study in the insurance industry », Information Systems Research, vol. 1, no 4, décembre, p. 377-393.

Vitale, M.R., B. IVES et C.M. BeATH (1986), "Linking information technology and corporate strategy: an organizational view», Comptes rendus de la $7^{e}$ International Conference on Information Systems, San Diego, California, 15-17 décembre, p. 265-276.

Wiseman, C. (1988), Strategic Information Systems, Homewood, Illinois, Irwin. 